\title{
Selección de pruebas del lenguaje y análisis crítico de su aplicación en población infantil con discapacidad auditiva
}

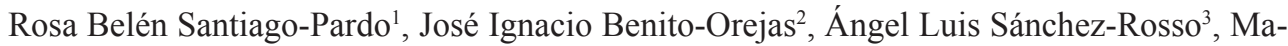 \\ ría Isabel Rico-Paino ${ }^{4}$, Ana Herrero-Galiacho ${ }^{5}$, y Lucía Isabel de Castro ${ }^{6}$
}

Recibido: 3 de diciembre de 17 / Revisado: 15 de marzo de 2018 / Aceptado: 4 de mayo de 2018.

Resumen. El objetivo de este trabajo consiste en seleccionar aquellas pruebas que mejor permitan examinar, desde un punto de vista formal, el lenguaje del niño con hipoacusia. Dado que el desarrollo lingüístico de las personas con discapacidad auditiva no avanza de forma homogénea en todas sus dimensiones, consideramos fundamental analizar todos los aspectos del lenguaje. Las pruebas escogidas, según criterios fijados en nuestro estudio, han sido: PPVT-III Peabody. Test de Vocabulario en Imágenes; Evaluación fonológica del habla infantil (L. Bosch); Registro Fonológico Inducido (M. Monfort); Evaluación de la Discriminación Auditiva y Fonológica (EDAF); Escala Magallanes de Lectura y Escritura (EMLE-TALE-2000); Prueba de Lenguaje Oral de Navarra-Revisado (PLON-R); Batería de Lenguaje Objetiva y Criterial Screening-Revisado (BLOC S-R); Test de Comprensión de Estructuras Gramaticales (CEG), y el Subtest de Vocabulario del WISC-IV. Esta evaluación se complementa con un cuestionario dirigido a quienes aplican los test y con la opinión de expertos logopedas en el terreno práctico. Como resultados, en general, todas las pruebas se han mostrado adecuadas según su finalidad. Las que mejor se corrigen son EDAF, PPVT-III Peabody y BLOC-SR y la que peor, la Evaluación fonológica del habla infantil de Laura Bosch. Para nosotros, el procedimiento de aplicación de PPVT-III Peabody y PLON-R es ideal para niños con hipoacusia, presentando mayores dificultades el EDAF y BLOC-SR y siendo más difíciles de aplicar, el Registro fonológico de Laura Bosch y el EMLE-TALE-2000. Consideramos que esta selección de pruebas es adecuada, siempre y cuando tengamos en cuenta la edad de desarrollo del niño con discapacidad auditiva y las modificaciones específicas que debemos adoptar en esta población.

Palabras Clave: Cribado auditivo neonatal; Pérdida auditiva; Diagnóstico precoz; Desarrollo del lenguaje infantil; Pruebas de lenguaje; Cuestionario.

[en] Selection of tests of language and critical analysis of its application in children with hearing impairment

\footnotetext{
Departamento de Pedagogía y Grado en Logopedia de la Facultad de Medicina. Universidad de Valladolid. España. santiago@med.uva.es

2 Servicio de Otorrinolaringología del Hospital Clínico Universitario de Valladolid. España.

3 Departamento de Psicología y Grado en Logopedia de la Facultad de Medicina. Universidad de Valladolid. España.

4 Grado en Logopedia de la Facultad de Medicina. Universidad de Valladolid. España.

5 Grado en Logopedia de la Facultad de Medicina. Universidad de Valladolid. España.

6 Grado en Logopedia de la Facultad de Medicina. Universidad de Valladolid. España.
} 


\begin{abstract}
The objective of this study has been to select the tests that allow us to study in the best possible way - from a formal point of view - the different aspects of the language of the child with hearing loss. Although there is not a consensus, we consider that in the case of a child with hearing loss, it is essential to analyze all aspects of the language. This will provide us a deep knowledge of their language development, which it is not homogenous in all areas. The language tests that we chose and analyzed were: PPVT-III Peabody. Test de Vocabulario en Imágenes; Evaluación fonológica del habla infantil (L. Bosch); Registro Fonológico Inducido (M. Monfort); Evaluación de la Discriminación Auditiva y Fonológica (EDAF); Escala Magallanes de Lectura y Escritura (EMLE-TALE-2000); Prueba de Lenguaje Oral de Navarra-Revisado (PLON-R); Batería de Lenguaje Objetiva y Criterial Screening-Revisado (BLOC S-R); Test de Comprensión de Estructuras Gramaticales (CEG), y el Subtest de Vocabulario del WISC-IV. This assessment is complemented by a questionnaire addressed to those who perform tests and the opinion of expert speech therapists in the practical field. In general terms, all the tests have been appropriate depending on their purpose. The tests that are corrected better are EDAF, PPVT-III Peabody and BLOC-SR. The test that is corrected worse is the phonological record of Laura Bosch. For us, the procedure of application of PPVT-III Peabody and PLON-R is ideal for children with hearing loss, presenting major difficulties the EDAF and BLOC-SR and being very difficult to implement, the phonological record of Laura Bosch and the EMLE-TALE-2000. We believe that the selected tests are enough to evaluate the development of the language in the child with hearing impairment, when we apply them correctly according to the age of development and take into account the specific modifications that we adopt in this population.
\end{abstract}

Key words: Neonatal hearing screening; Hearing loss; Early diagnosis; Child language development; Language test; Questionnaire.

Cómo citar: Santiago-Pardo, R.B., Benito-Orejas, J.I., Sánchez-Rosso, Á.L., Rico-Paino, M.I., Herrero-Galiacho, A., y de Castro, L.I. (2018). Selección de pruebas del lenguaje y análisis crítico de su aplicación en población infantil con discapacidad auditiva. Revista de Investigación en Logopedia, $8(2), 147-164$.

\title{
Introducción
}

La hipoacusia es la deficiencia sensorial más frecuente, pues afecta a unos tres por mil de recién nacidos (RN) (Benito-Orejas, Ramírez, Morais, Fernández-Calvo y Almaraz, 2008) manifestándose desde el nacimiento (congénita) en un $80 \%$ de los casos (Benito-Orejas y Silva-Rico, 2013). En la mayoría de los pacientes uno de los progenitores es oyente (Fitzpatrick, Angus, Durieux-Smith, Graham y Coyle, 2008) lo que explica que estas familias quieran que sus hijos desarrollen el lenguaje oral (Vohr et al., 2008). La sordera impacta negativamente en el desarrollo y la salud del niño durante toda su vida, influyendo no sólo en el lenguaje, sino también en las funciones mentales, sociales y cognitivas, en los logros educativos y en las oportunidades socio-económicas y de empleo laboral (Mohr et al., 2000), suponiendo un importante coste a la sociedad (Schroeder et al., 2006).

A través de la audición, el niño aprende el habla "por imitación" y por medio de éste, desarrolla la comunicación y adquiere conocimiento sobre su entorno (Northern y Downs, 1981). Aunque el órgano auditivo ha madurado antes del nacimiento, las conexiones nerviosas aferentes y la corteza auditiva sólo se desarrollan morfológica y funcionalmente bajo la influencia del estímulo sonoro y, además, como otras funciones biológicas, con una precisa cronología. La adquisición del lenguaje depende de un "periodo crítico" comprendido entre el nacimiento y los 3 años aproximadamente. Un niño que no tenga contacto con el sonido durante este tiempo, nunca logrará la competencia lingüística acorde con su potencial (Kral y O'Donoghue, 2010). 
La identificación precoz, el diagnóstico y el seguimiento de la hipoacusia infantil resultan fundamentales para iniciar el proceso de re/habilitación, lo que facilitará al niño sordo recibir la máxima información auditiva durante el período crítico de desarrollo del lenguaje hablado, reduciendo los efectos de la deprivación sonora.

La evaluación forma parte del proceso de intervención porque permite observar la evolución y controlar su efectividad (Ruiz-Paredes, 2011). En la literatura observamos que las pruebas de lenguaje destinadas a oyentes han tenido que adaptarse a la población con déficit auditivo, y se han utilizado sobre todo, más que para un diagnóstico formal del lenguaje, para valorar la evolución de una adaptación protésica y, especialmente, de un implante coclear (Manrique-Rodríguez y Huarte-Irujo, 2013; Manrique et al., 2004; Svirsky, 2002), pues el interés inicial era conocer el efecto que la innovadora tecnología producía en el desarrollo del lenguaje (Lederberg, Schick y Spencer, 2012).

Aunque analizaremos el progreso madurativo general y los umbrales de audición en el niño con hipoacusia, es fundamental el aspecto lingüístico, para cuya estimación disponemos de diferentes métodos. Unos de carácter formal y de tipo prescriptivo (estandarizado y basado en el razonamiento deductivo) que son los que nosotros emplearemos y otros menos formales o alternativos, más descriptivos (derivados del razonamiento inductivo) que, como señalaremos posteriormente, son los que, por su carácter funcional, se utilizan con más frecuencia en la clínica logopédica.

La preocupación por investigar el lenguaje, surge a mediados del siglo XX con los escritos de Johnson, Darley y Spriestersbach en 1963, donde se explican diversos métodos de diagnóstico para categorizar el habla. Poco después, se introduce el concepto de asociaciones internas o mediadores, con la aparición en 1968 del "Test Illinois de Habilidades Psicolingüísticas" (ITPA) (Kirk, McCarthy y Kirk, 2004) y a partir de aquí, los objetivos para evaluar el lenguaje se basan en influencias tanto lingüísticas como psicológicas, dando importancia a la morfosintaxis, a las relaciones y rasgos semánticos, al nivel cognitivo y a la calidad del uso del lenguaje. Es en los años ochenta cuando aparece el concepto de la pragmática, creando un cambio en el significado de la evaluación del lenguaje, al incluir características emocionales, sociales y personales.

Actualmente, disponemos de numerosas pruebas para estimar los diferentes aspectos del lenguaje y sin embargo de pocas exploraciones específicas para las personas con discapacidad auditiva, con excepción del test Carolina Picture Vocabulary Test (CPVT) (Kline y Sapp, 1989), que valora la comprensión del vocabulario emitido con la lengua de signos, el test de Evaluación de la Discriminación Auditiva y Fonológica (EDAF) (Brancal, Alcantud, Ferrer y Quiroga, 2009) y la Valoración de la Percepción Auditiva: explorando los sonidos y el lenguaje (Gotzens y Marro, 2001). Como consecuencia de la escasez de recursos lingüísticos aplicables en caso de hipoacusia, se emplean aquellas pruebas que por sus características puedan trasladarse a este colectivo de personas, con diversas adaptaciones para su correcta evaluación. Entre las más utilizadas destacan: Test de Análisis Gramatical del Lenguaje Provocado de nivel Preoperacional (GAEL-P) (Moog, Kozak y Geers, 1987), Test de Vocabulario en Imágenes PPVT-III Peabody (Dunn, Dunn y Arribas, 2010), Test de Vocabulario de Boston (García, Sánchez y Delviso, 1986; Goodglass y Kaplan, 1986), Batería de Lenguaje Objetiva y Criterial (BLOC) (Puyuelo-Sanclemente, Renom-Pinsach, Solanas-Pérez y Wiig, 2007), Test Illinois de Aptitudes Psicolingüísticas (ITPA) (Kirk, McCarthy y Kirk, 2004), Cuestionario de Análisis de Retraso del 
Lenguaje (AREL) (Pérez y Serra, 1998), Prueba de Lenguaje Oral de Navarra Revisado (PLON-R) (Aguinaga, Armentia, Fraile, Olangua y Uriz, 2004), Desarrollo de la Morfosintaxis en el Niño (TSA) (Aguado-Alonso, 1989), Test de Comprensión de Estructuras Gramaticales (CEG) (Mendoza, Carballo, Muñoz y Fresneda, 2005; Calet-Ruiz, Mendoza-Lara, Carballo-García, Fresneda-López y Muñoz-López, 2010), Test de Habilidades Metalingüísticas (THM) (Gómez, Valero, Buades y Pérez, 2005), Exploración del Lenguaje Comprensivo y Expresivo (ELCE) (López-Ginés et al., 1997; López-Ginés et al., 2007), Escala Magallanes de Lectura y Escritura (EMLE-TALE-2000) (Toro-Trallero, Cervera-Laviña y Urío-Ruiz, 2002), Registro Fonológico Inducido (RFI) (Monfort y Juárez, 1989), Evaluación fonológica del habla infantil de Laura Bosch (Bosch-Galcerán, 2004) y la Escala verbal del WISC-R (Escala de Inteligencia de Wechsler para niños) (Wechsler, 2005).

La elección y uso de estas pruebas debe ser selectiva, pues en la mayoría predomina el componente verbal y los resultados obtenidos podrían estar mediatizados por las características de la hipoacusia. Como luego veremos, incluso en pruebas manipulativas se pueden ver alterados los resultados, como consecuencia del contenido verbal de las instrucciones de la propia prueba y la necesaria mediación verbal (función mediadora del lenguaje) para resolver algunos de los problemas que en ellas se plantean. Es preciso acondicionarlas al tipo de paciente (utilizar apoyos visuales para facilitar la comprensión de las instrucciones, repetir las explicaciones o complementarlas con un vocabulario adaptado y más sencillo, adecuar los tiempos indicados para la aplicación...) o utilizar pruebas sin contenido verbal.

El objetivo de este trabajo ha consistido por una parte en seleccionar y describir las pruebas con las que vamos a estudiar de forma prescriptiva y formal los diferentes aspectos del lenguaje de los niños con hipoacusia prelocutiva y, por otra, en analizar desde un punto de vista subjetivo, aunque contrastado con otros profesionales, algunas de sus características, utilizando cuestionarios para recabar dicha información.

Es importante resaltar que este estudio no ha pretendido analizar el desarrollo de las aptitudes y características previas a la adquisición del lenguaje, sino determinar el grado de "competencia lingüística" (entendido como el dominio del léxico, reglas, estructuras y funciones del lenguaje) puesto de manifiesto en un momento determinado de la evolución de las personas con discapacidad auditiva. Como consecuencia, comenzamos la evaluación a partir de los 3 años de edad, al considerar que es cuando el niño tiene unos mínimos de competencia lingüística, siendo la experiencia anterior a esta edad demasiado frágil. Esto explica que no se haya utilizado ninguna prueba que evalúe aptitudes psicolingüísticas ni repertorios previos a la adquisición y desarrollo del lenguaje, aplicándose exclusivamente aquellas que lo valoran en sus dimensiones convencionales, a saber: fonológico-fonética, léxico-semántica, morfológica, sintáctica y pragmática.

Finalmente podremos concluir, si esta metodología resulta para nuestros propósitos, fiable y aplicable clínicamente. 


\section{Método:}

\section{Participantes:}

El tema de este documento está encuadrado en el proyecto de investigación multidisciplinar iniciado en agosto de 2015: "Desarrollo del lenguaje en niños con detección temprana de hipoacusia neonatal", donde participan logopedas, estudiantes de Logopedia, profesores de los departamentos de Pedagogía y Psicología del Grado en Logopedia de la Universidad de Valladolid (UVa) y un especialista en el área de Otorrinolaringología del Hospital Clínico Universitario de Valladolid (HCU-VA) (Benito-Orejas et al., 2017).

Los participantes del estudio son aquellos con hipoacusia neonatal o prelocutiva bilateral, que hayan sido diagnosticados en estos últimos 15 años en el HCU-VA, con edades comprendidas entre los 3 y los 15 años y con los criterios de inclusión/ exclusión expuestos en un trabajo anterior (Benito-Orejas et al., 2017), con lo que hemos obtenido finalmente una muestra de 45 participantes.

Además, los propios miembros del equipo de investigación, logopedas, así como los padres y madres de los niños estudiados han podido participar de manera activa en este estudio.

\section{Material / Instrumentos:}

Para evaluar la competencia lingüística de nuestros casos a estudiar, se aplicaron diferentes pruebas en función de la edad, cuya selección se ha llevado a cabo atendiendo a los siguientes criterios:

1. Estar estandarizadas y en español (idioma principal de los participantes). Para que los resultados sean objetivos, deben disponer de una validez demostrada y baremada.

2. Ofrecer la posibilidad de obtener los resultados en unidades centiles y/o típicas, a fin de poderlos unificar con el resto de pruebas y permitir comparaciones basadas en una misma medida.

3. Ser sencillas a la hora de estudiar, aplicar y corregir. Este criterio es subjetivo y el propio equipo decidirá si una prueba cumple o no estos requisitos.

4. No ser excesivamente extensas. De nuevo, una norma subjetiva, cuya finalidad pretende evitar en los niños la fatiga y la consiguiente invalidez de la prueba, razón por la cual cuando era necesario aplicar alguna de las pruebas de mayor duración (concretamente el BLOC-SR y el EMLE-TALE 2000), la exploración se realizaba en dos sesiones procurando que ninguna de ellas sobrepasara los 60 minutos de duración.

5. Que el rango de aplicación sea amplio y, a ser posible, que abarque población comprendida entre los 3 y los 15 años de edad. De no ser factible, se buscarían pruebas similares cuya combinación cubra el rango de edad de la población a estudiar, aunque la evaluación con la misma prueba es preferible, al ofrecer mayor objetividad a la hora de comparar los resultados.

6. De entre las pruebas escogidas, se elegirán finalmente aquellas de las que se disponga y de las que, por haber sido utilizadas, se tenga una mayor experiencia. 
A partir de estos criterios específicos, se seleccionaron las pruebas que nos iban a permitir recoger información sobre la dimensión fonológico-fonética (que analiza las formas articulatorias y de emisión de fonemas y el grado de conocimiento que el participante tiene de los rasgos que diferencian unos fonemas de otros); la semántica (estudia el significado, de palabras aisladas, primero con definiciones obvias y concretas y luego más complejas y abstractas; pero también se refiere a la función de las palabras y refleja la comprensión de relaciones entre objetos, acciones y eventos); la morfología (analiza la estructura y forma de las palabras, incluyendo, por ejemplo, los marcadores gramaticales que se utilizan para indicar el plural o los tiempos verbales) (Svirsky, 2002); la sintaxis (se ocupa de las reglas mediante las cuales se combinan palabras y otros elementos de la estructura de una oración para formar frases gramaticalmente correctas); y la pragmática (se refiere a las reglas de intercambio comunicativo, ordenadas de acuerdo a una jerarquía. Las más elementales aluden a la comunicación no verbal e incluyen, por ejemplo, el empleo apropiado del contacto visual, la expresión facial, el uso de gestos; mientras que las más complejas involucran la función exclusiva de la comunicación verbal). Además, hemos considerado la conveniencia de disponer de otras dos pruebas para evaluar dos aspectos más que nos han parecido interesantes:

Por un lado, una prueba de discriminación auditiva que nos informe, no ya de la audición de los niños, sino de su capacidad para discriminar los sonidos del ambiente $y$, fundamentalmente, los sonidos del habla, pues entendemos que un déficit en discriminación auditiva podría explicar algunas de las dificultades fonoarticulatorias que podemos comprobar en esta población.

Por otro lado, una prueba de lectura y escritura a niños mayores de 6 años, con la idea de comprobar hasta qué punto existe una correlación entre el nivel de competencia lingüística encontrado en la lengua oral y el dominio que esos mismos niños puedan poner de manifiesto en lenguaje escrito.

La segunda parte de este trabajo consta de varios cuestionarios. El primero de ellos recoge la opinión de los logopedas participantes en el proyecto, respecto a determinados aspectos subjetivos de las seis pruebas finalmente analizadas: Test de Vocabulario en Imágenes PPVT-III Peabody, Evaluación fonológica del habla infantil de Laura Bosch, EDAF, EMLE-TALE-2000, PLON-R y BLOC-SR. Para obtener estos resultados se distribuyó el siguiente cuestionario de cuatro preguntas a los quince logopedas responsables de la realización de las pruebas:

1. ¿Cree que esta prueba es adecuada para ser utilizada en niños/as con hipoacusia? ¿Por qué?

2. ¿Considera que es fácil la corrección del test? ¿Por qué?

3. ¿Le resulta adecuado el método de aplicación? ¿Por qué?

4. ¿Realizaría algún tipo de modificación? ¿Cuál?

Así mismo a los padres/madres y a los niños evaluados mayores de 12 años, se les ofreció un cuestionario general de satisfacción, que respondieron libremente. Respecto a la aplicación de las pruebas, las principales preguntas formuladas con carácter exclusivo a los padres/madres, fueron:

1. ¿Cómo le resultó el proceso de aplicación de las pruebas del lenguaje a su hijo/a? ¿Por qué? 
2. El tiempo empleado en la realización de las pruebas, ¿cómo lo considera? ¿Por qué?

3. ¿Qué opina sobre la realización de este tipo de estudios?

4. Aspectos que cree se podrían mejorar.

Por otra parte, se elaboró también un cuestionario de satisfacción para que fuera contestado por los niños que participaron en el estudio, con más de 12 años, al considerar que a partir de esa edad pueden tener criterio para dar su opinión sobre el proceso de evaluación que se les aplicó. Con ello se pretendió, además, que se sintieran protagonistas hasta el final del proceso. Las preguntas realizadas fueron las siguientes:

1. ¿Se te hizo muy largo el tiempo que estuviste con las pruebas?

2. ¿Te acuerdas de alguna prueba que fuera más difícil de entender, muy larga o aburrida?

3. Para realizar las pruebas, ¿preferirías estar más tiempo sólo 1 día, o estar menos tiempo y venir más días?

4. Cuéntanos lo que más y lo que menos te ha gustado.

Por último, se contactó con 15 centros profesionales de Logopedia privada, en la ciudad donde se desarrolla el proyecto, para conocer sus preferencias respecto a las pruebas que utilizan en personas con discapacidad auditiva a fin de poder realizar comparaciones con las escogidas en este estudio. Para ello, se les envió vía telemática el siguiente cuestionario:

1. ¿Qué pruebas utiliza para evaluar el lenguaje en niños/as con discapacidad auditiva en edad escolar? y ¿por qué?

2. ¿Cuál de las que emplea, considera que es más apropiada para este colectivo?

3. ¿Qué modificaciones realizaría, si las hubiera, en las pruebas para evaluar el lenguaje en niños/as con discapacidad auditiva en edad escolar?

\section{Procedimiento y resultados:}

\section{Pruebas de lenguaje:}

A continuación, referimos las pruebas utilizadas en el Proyecto de Investigación anteriormente mencionado y los aspectos del lenguaje que valoran:

- "PPVT-III Peabody. Test de Vocabulario en Imágenes": dimensión semántica, aspecto comprensivo (Dunn, Dunn y Arribas, 2010).

- "Evaluación fonológica del habla infantil" de Laura Bosch: dimensión fonológico-fonética (Bosch Galcerán, 2004).

- "Registro Fonológico Inducido (RFI)": dimensión fonológico-fonética (Monfort y Juárez, 1989).

- "Evaluación de la Discriminación Auditiva y Fonológica (EDAF)": área de discriminación auditiva (Brancal, Alcantud, Ferrer y Quiroga, 2009). 
- "Escala Magallanes de Lectura y Escritura (EMLE- TALE-2000)": modalidad de lenguaje escrito, lectura y escritura (Toro, Cervera y Urío, 2002).

- "Prueba de Lenguaje Oral de Navarra-Revisado (PLON-R)": dimensiones fonológico-fonética, morfosintáctica, semántica y pragmática (Aguinaga, Armentia, Fraile, Olangua y Uriz, 2004).

- "Batería de Lenguaje Objetiva y Criterial Screening-Revisado (BLOC S-R)": dimensiones semántica, morfosintáctica y pragmática (Puyuelo, Renom, Solanas y Wiig, 2007).

- "Test de Comprensión de Estructuras Gramaticales (CEG)": dimensión morfosintáctica, aspecto comprensivo (Mendoza, Carballo, Muñoz y Fresneda 2005).

- "Escala de Inteligencia WECHSLER para niños (WISC-IV)". Escala verbal, prueba de Vocabulario: dimensión semántica, aspectos compresivo y expresivo (Wechsler, 2005).

Las características fundamentales de cada prueba se resumen en lo siguiente:

1. Test de Vocabulario en Imágenes Peabody PPVT-III, con adaptación al castellano y baremación. Evalúa comprensión de vocabulario. El examinador dice un listado de palabras y el evaluado debe señalar, entre varias opciones, la imagen que se corresponde con cada una de las palabras propuestas por el examinador. No requiere respuestas verbales. Duración aproximada de 20 minutos.

2. Evaluación fonológica del habla infantil de Laura Bosch: analiza los patrones de producción del habla. Consiste en un análisis fonológico a partir de 32 palabras, que incluyen todos los fonemas del castellano. En su aplicación se muestran láminas de dibujos en las que se ven imágenes y acciones acompañadas de palabras, que el evaluado debe pronunciar. Se registra el número de errores cometidos y los fonemas equívocos. Destinada para niños de entre 3 y 7 años (edad en la que el niño suele haber completado el proceso de adquisición del repertorio fonológico del castellano). Duración aproximada de 20 minutos.

3. Registro Fonológico Inducido (RFI): recoge las peculiaridades del habla del niño. Repasa el repertorio fonológico fundamental en castellano, en producción inducida o en repetición si fuera necesario. La valoración es cualitativa y cuantitativa (comparándola con la media de errores de articulación obtenida en un grupo de niños de la misma edad). Exige respuesta verbal, pero no de lectura. Se aplica entre 3 y 6 años y medio. Tiene una duración de 15-20 minutos.

4. Evaluación de la Discriminación Auditiva y Fonológica (EDAF): sirve para valorar alteraciones en la discriminación auditiva y fonológica a través de 5 subtest: discriminación de sonidos del medio (DSM), discriminación figura-fondo (DFF), discriminación fonológica en palabras (DFP), discriminación fonológica en logotomas (DFL) y memoria secuencial auditiva (MSA). Utiliza como material un libro de imágenes y un disco compacto (CD) con la grabación de los estímulos sonoros correspondientes. Se aplica a partir de los 3 años, y su duración es variable según las habilidades y capacidades del niño, pero se estima de media entre 30 y 45 minutos. 
5. Escala Magallanes de Lectura y Escritura (EMLE-TALE-2000): con esta prueba se pretenden conocer, de manera cualitativa y cuantitativa, la capacidad de lectura y escritura de los sujetos, analizando el nivel de adquisición de las habilidades de conversión grafema-fonema, la calidad lectora en voz alta, valorando la fluidez, la entonación y los tipos de errores cometidos tanto en lectura como en escritura (dictado y copia). Duración aproximada de 1 hora.

6. Prueba de Lenguaje Oral de Navarra - Revisado (PLON-R): explora el lenguaje oral en sus aspectos fonológicos, morfológicos, morfosintácticos, pragmáticos y léxicos, es decir, las funciones informativas, de petición, metalingüística y el lenguaje espontáneo. Exige respuesta verbal pero no de lectura. Destinada para niños de entre 3 y 6 años. Duración estimada de 35 minutos por cada subtest de edad.

7. Batería de Lenguaje Objetiva y Criterial (BLOC) Screening - Revisada (BLOC-SR): mide competencia lingüística en 4 dimensiones: morfología (flexión y formación de palabras), sintaxis (estructura de la oración), semántica (función significativa de elementos de la oración) y pragmática (uso del lenguaje en situaciones concretas). Cuenta con datos normativos (por lo que es una batería objetiva), pero también admite una valoración a partir de criterios de referencia (siendo así mismo “criterial”). Dispone del BLOC-INFO que permite la corrección de ambas baterías (reducida y completa) a través del ordenador. Aplicable entre los 5 y 14 años, el BLOC-SR tiene una duración aproximada de 1 hora.

8. Si en el screening del BLOC-SR se fallara especialmente en los aspectos gramaticales, aplicaríamos el Test de Comprensión de Estructuras Gramaticales (CEG): que valora la interpretación de las estructuras sintácticas (comprensión de construcciones gramaticales de diferente complejidad). No requiere respuesta verbal. Aplicable entre 4 y 11 años. Con una duración entre 15-20 minutos, según la edad. Establece una valoración cualitativa y cuantitativa.

9. Si los fallos registrados en el screening del BLOC-SR fueran en el plano semántico, utilizaríamos la Escala de Vocabulario del WISC-IV (Escala de Inteligencia WECHSLER para niños): el test de Vocabulario, integrado en la "Escala Verbal", evalúa la comprensión y expresión verbal, memoria y conocimientos adquiridos. El niño debe enunciar de forma oral el significado de las palabras que se le presentan. Aplicable entre los 5 y 16 años. El tiempo de realización es variable, en función de los test que se utilicen.

Para la aplicación de las pruebas se les indica a los niños que traigan colocados sus audífonos o implante coclear (IC). El desarrollo de cada examen se estima de acuerdo al procedimiento descrito en la propia prueba. Algunas de estas exploraciones se grabaron en video y/o audio, previo consentimiento paterno.

No se suministraron todos los ejercicios a todos los niños, teniendo en cuenta el rango de aplicación marcado por las propias pruebas, planificando la evaluación en función de la edad, de sus características y de los estudios realizados previamente. A los niños de la misma edad se les estudió con las mismas pruebas. Así, p. ej., a los de edad comprendida entre los 3 y 6 años se les aplicó el PLON-R para evaluar las distintas dimensiones del lenguaje, en tanto que en los mayores de 6 años se utilizó el BLOC-SR para ese mismo propósito. De la misma manera, únicamente se administró el EMLE-TALE-2000 a los mayores de 6 años, por entender que es a partir 
de esta edad cuando el niño tiene ya un cierto dominio de los procesos de lectura y escritura, excluyendo a aquellos niños cuyos padres habían manifestado en la entrevista que todavía no sabían leer/escribir, aunque fueran mayores de dicha edad.

Finalmente, de las nueve pruebas mencionadas en el apartado anterior, fueron seleccionadas para su análisis en este trabajo, por su mayor frecuencia de uso en la población estudiada, las seis siguientes: PPVT-III Peabody, Evaluación fonológica del habla infantil de Laura Bosch, EDAF, EMLE-TALE-2000, PLON-R y BLOC-SR.

\section{Resultado de los cuestionarios:}

La carencia en lengua castellana de pruebas de lenguaje específicamente diseñadas para la población sorda, hizo que nos planteáramos evaluar los pros y contras que la aplicación de las seleccionadas podía tener en la muestra objeto de este estudio. Para ello, una de los componentes del grupo de trabajo, llevó a cabo un Trabajo Fin de Grado (TFG) en el que, con la colaboración del resto de miembros del equipo, se planteó utilizar dos cuestionarios, uno dirigido a los logopedas del Proyecto y otro a un conjunto de logopedas de centros de Logopedia privada, de nuestro entorno (Rico-Paino, 2016). Por otra parte al finalizar la transmisión de los resultados a las familias, se les entregó un cuestionario de satisfacción y otro a los participantes con más de 12 años.

Los resultados obtenidos fueron los siguientes:

\section{Del cuestionario a los logopedas evaluadores en el proyecto:}

Tras analizar las opiniones de los once logopedas participantes en el proyecto, que utilizaron las seis pruebas seleccionadas (PPVT-III Peabody, Evaluación fonológica del habla infantil de Laura Bosch, EDAF, EMLE-TALE-2000, PLON-R y BLOC-SR), las respuestas recogidas se resumen como sigue:

1. Adecuación de las pruebas a su finalidad: todas las pruebas se consideran adecuadas para ser utilizadas en niños con discapacidad auditiva, cada una con la finalidad con la que ha sido creada. La más criticada fue el BLOC-SR, por ser excesivamente larga y producir fatiga en algunos casos.

2. Corrección de las pruebas: EDAF, PPVT-III Peabody y BLOC-SR se corrigen con facilidad. La prueba de EMLE-TALE-2000 resulta difícil de calificar en un 40\% de los encuestados. Al ser la corrección del EMLE-TALE-2000 mecanizada, el único dato objetivo que se nos suministra es la puntuación obtenida en "comprensión lectora", de forma que la competencia alcanzada en otros aspectos, como la lectura mecánica, escritura espontánea y dictado, ha de ser sometida a una evaluación de tipo criterial, en función del número de errores cometidos en cada una de las pruebas. El PLON-R es en general sencilla de registrar, pero al ser una prueba de screening permite muy escaso margen de error (basta fallar 1 ítem, teniendo bien los otros 5, para que no se puntúe).

3. Método de aplicación de las pruebas: PPVT-III Peabody y PLON-R tienen un correcto método de aplicación para los niños con hipoacusia, aunque en el PPVT-III Peabody hay palabras complejas de ser comprendidas en determinadas franjas de edad. El EDAF tiene el inconveniente de que, al ser parte de la prueba grabada en disco compacto $(\mathrm{CD})$, provoca un ruido de fondo que dificulta la comprensión en 
niños con hipoacusia, especialmente menores de 6 años. El BLOC-SR muestra una duración excesiva y una gran complejidad en la forma de expresar las consignas, lo que pudiera haber influido en los bajos resultados obtenidos en el apartado de "morfología". La Evaluación fonológica del habla infantil de Laura Bosch inducía a equívocos en frecuentes ocasiones. El EMLE-TALE-2000 solía resultar monótono y largo para algunos niños, en especial si a la discapacidad auditiva se sumaba un déficit de atención.

En resumen, el resultado obtenido tras este análisis indica, que ninguna de las pruebas evaluadas ha sido considerada perfecta por los logopedas que las han utilizado, en cualquiera de los tres aspectos analizados. En la Figura 1 se representa en colores la opinión de los miembros del equipo de logopedas del proyecto a tres preguntas que deben responder por cada una de las 6 pruebas indicadas: si se considera la prueba "adecuada" para niños con hipoacusia, si creen que se "corrige" con facilidad y finalmente si su "aplicación" es sencilla en esta población infantil. Tal y como se aprecia en la Figura 1, donde el color verde indica un acuerdo total con la pregunta planteada, el azul parcial y el rojo manifiesta desacuerdo, PPVT-III Peabody y PLON-R son las que obtienen mejores puntuaciones globales y la Evaluación fonológica del habla infantil de Laura Bosch y el BLOC-SR, las peores.

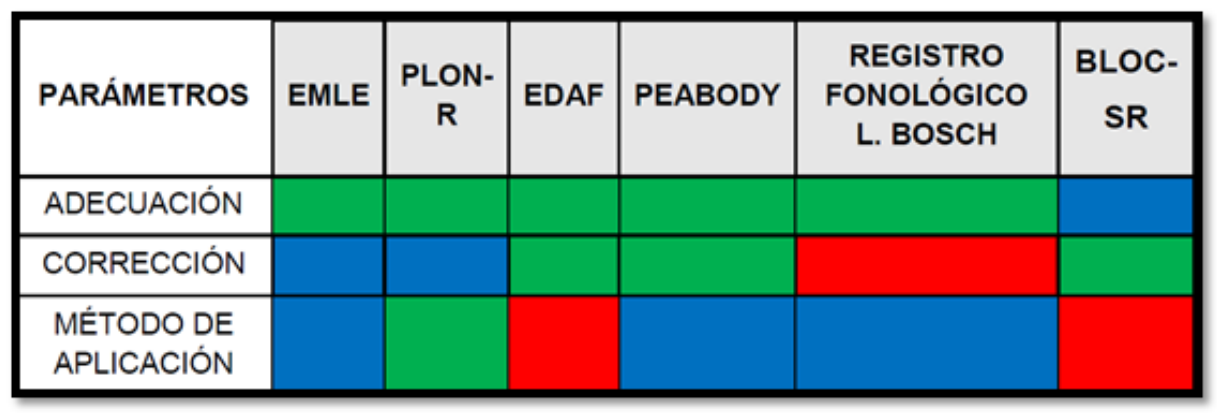

Figura 1: Opinión de los logopedas. Fuente Rico-Paino, 2016.

Pero además de las pruebas escogidas para el estudio del lenguaje y de sus características, se deben tener también en cuenta las cualidades propias del explorador, pues al tratarse de población infantil, pueden influir de forma importante en los resultados. La personalidad, la actitud, la experiencia y el buen uso de refuerzos que faciliten al niño disfrutar de las actividades, permitirán obtener el máximo rendimiento (Rico-Paino, 2016). Para evitar en lo posible el sesgo, se constituyeron varios equipos de logopedas jóvenes, a fin de que estas características quedaran equiparadas entre los distintos miembros del grupo.

\section{Del cuestionario de satisfacción a las familias participantes:}

Aproximadamente 1/3 de las familias contestaron a este cuestionario, que fue distribuido tras explicarles el resultado de las pruebas y al que respondieron libremente y de forma anónima por correo. En general, consideraban importante la realización de este tipo de estudios, aunque su queja principal era el tiempo excesivo utilizado en su aplicación (unas dos horas, 1-2 días). Al plantearles reducir la duración de las 
sesiones, aumentando su número, también encontraban inconvenientes. Por tanto, es muy importante saber escoger aquellas pruebas que ofrezcan un máximo rendimiento en el menor tiempo posible, lo que con frecuencia no resulta un problema sencillo de resolver.

\section{Del cuestionario a logopedas de centros profesionales:}

De los quince centros profesionales de Logopedia privada a los que se envió telemáticamente el cuestionario, respondieron once y en los cuatro restantes expresaron que, en su unidad, no se realizaban evaluaciones a niños con hipoacusia. Las respuestas obtenidas a las cuestiones planteadas fueron las siguientes:

1. Las pruebas que aplican los logopedas profesionales entrevistados para evaluar el lenguaje de los niños con hipoacusia son muy variadas, pero en general, la mayoría coinciden con las utilizadas en este proyecto, destacando por su frecuencia de uso, el PPVT-III Peabody, EMLE-TALE-2000, EDAF y PLON-R. Aunque se emplean pruebas estándar, su uso se adecúa a las necesidades. Más que una exploración formal, se pretende una evaluación funcional (metodología descriptiva) de los diferentes niveles de lenguaje que permita su aplicación inmediata en la orientación de la terapia re/habilitadora.

2. En ninguno de los centros consultados utilizan una única prueba para evaluar el lenguaje de los niños con pérdida auditiva. Todos están de acuerdo que para obtener la información necesaria se precisan diferentes test que varían en función de cada niño, dada la heterogeneidad que caracteriza a esta población. Las pruebas a aplicar deben adaptarse a la edad, al grado de pérdida auditiva, al uso de medios protésicos, a la fase del proceso de re/habilitación y a la presencia de niños signantes, para quienes es muy difícil encontrar pruebas "adecuadas".

3. Finalmente, se pregunta a los logopedas profesionales las modificaciones que realizarían en las pruebas del lenguaje utilizadas en este colectivo de niños con discapacidad auditiva. Todos consideran la necesidad de que las pruebas se puedan adaptar al estilo comunicativo del niño sordo; usando el soporte de la lectura labial, los apoyos visuales, la comunicación bimodal, la lengua de signos, etc. También resaltan la importancia de poder conocer el desempeño auditivo que estos niños manifiestan en otros contextos (cuya información quizá pudiera recogerse a través de cuestionarios a padres y profesores).

Vale la pena incidir en la diferencia que existe entre una evaluación "funcional" y una evaluación "formal". El objetivo de la evaluación funcional es detectar necesidades específicas que permitan diseñar un programa de intervención logopédica a un niño en concreto y en cuyo caso cabrían las adaptaciones mencionadas. Sin embargo, para una evaluación "formal", con una finalidad diagnóstica, se recomienda utilizar pruebas objetivas, debidamente baremadas y estandarizadas, en las que no cabría ningún tipo de adaptación (si bien, podría ser defendible utilizar la lectura labial), que iría en contra de la objetividad necesaria, tanto en su aplicación (incluidos los materiales y las consignas) como en su corrección, puntuación e interpretación de los resultados. Si la evaluación formal no tiene en cuenta estas premisas, no se pueden 
establecer comparaciones con los datos estándares de la población de referencia con la que han sido baremadas las pruebas.

\section{Discusión:}

Este proyecto nos brinda la oportunidad de poder estudiar desde el punto de vista lingüístico a un colectivo de 45 niños con diferentes grados de hipoacusia, a los que se ha realizado un diagnóstico, intervención y seguimiento protocolizados (Benito-Orejas et al., 2008; Benito-Orejas et al., 2016; Junta de Castilla y León, 2004) con unas características uniformes, aunque contemplando algunas excepciones que nos permitirán estudios comparativos posteriores.

Constituir un grupo multidisciplinar con diferentes especialistas de la audición, la Logopedia, la Psicología y la Pedagogía, permite obtener una visión más completa de los distintos aspectos que determinan las características del niño sordo, facilitando la interpretación de los resultados.

Desde hace más de 15 años y siguiendo las recomendaciones del Joint Committee on Infant Hearing (1982), comenzamos a aplicar un programa de cribado en nuestro hospital, con carácter universal (Benito-Orejas et al., 2008; Martínez, Benito, Condado, Morais y Fernández-Calvo, 2003). De manera coincidente, el desarrollo tecnológico ofreció la disponibilidad de audífonos digitales que permitieron rectificar el perfil auditivo desde las hipoacusias leves a las severas, enriqueciendo además la calidad de señal. Con la introducción del implante coclear (IC), se brinda finalmente la posibilidad de que un niño con sordera profunda acceda al lenguaje oral. A lo largo de este tiempo las mejoras han ido sucediéndose y la intervención ha podido ser cada vez más precoz. La regla internacional del "plan 1-3-6" (cribado auditivo en el primer mes, diagnóstico en los 3 primeros meses e intervención antes del sexto mes) que guía los programas de hipoacusia neonatal (Junta de Castilla y León, 2004; American Academy of Pediatrics, 1999; Joint Committee on Infant Hearing, 2007; CODEPEH (Comisión para la Detección Precoz de la Hipoacusia, 2010), conlleva el que el audioprotesista y el logopeda se enfrenten por primera vez en la historia, con niños diagnosticados de 3 meses, a los que se les debe iniciar un tratamiento. Los avances han sido tan espectaculares, que tal y como se ha demostrado, si esta intervención se produce en los 6 primeros meses de vida, se consigue que algunos niños alcancen unos niveles de lenguaje similares a los de los normoyentes a la edad de unos 5 años (Mayne, Yoshinaga-Itano, Sedey y Carey, 2000; Yoshinaga-Itano, Coulter y Thomson, 2000). Hemos pasado de trabajar con alumnos sordos profundos, en los que era preciso reeducar el lenguaje para llegar a un uso oral limitado, a encontrarnos en la actualidad con niños implantados tempranamente, que han conseguido una audición funcional, han desarrollado un lenguaje oral normalizado y se comunican y acceden a los aprendizajes del aula con ayudas y estrategias totalmente asumibles por los centros escolares ordinarios (Equipo de Audición y Lenguaje del CREENA, 2006).

La producción de la palabra y el lenguaje están directamente relacionados con la audición (hablamos como oímos), por lo que su análisis constituye una de las formas fundamentales para calibrar la evolución del niño con hipoacusia congénita o prelocutiva. Tal y como hemos comprobado a través de la entrevista realizada 
a las logopedas profesionales, la evaluación del lenguaje se considera básica para poder seguir, cuantitativa y cualitativamente el progreso del niño y detectar aquellas desviaciones que necesiten de una intervención especial. Pero también, para valorar un procedimiento de habilitación, describir el resultado de determinadas categorías e incluso con fines administrativos (Borg et al., 2002). Como quedó expuesto en la introducción, esta valoración se dirige a conocer la capacidad de comprensión y expresión de sonidos, palabras y frases, la inteligibilidad del habla, la extensión del léxico, el dominio de las estructuras morfosintácticas y la prevalencia del modo de comunicación audio-oral o gestual en la vida habitual del paciente (Molina-Hurtado y Huarte-Irujo, 2003).

Reconocida la importancia de la evaluación del lenguaje, nos planteamos qué pruebas deberíamos aplicar para realizar un análisis formal. Silva, Comerlatto Junior, Bevilacqua y Lopes-Herrera (2011) analizan la literatura, buscando los instrumentos que se utilizan habitualmente para estudiar el lenguaje de los niños con hipoacusia candidatos a IC y tras repasar las publicaciones de 5 años, detectan hasta 74 pruebas distintas (que incluyen test, cuestionarios e inventarios), lo que indica que son muchas las maneras que nos permiten realizar esta evaluación. Y a pesar de esta multiplicidad, la mayor parte de estudios se centran en el lenguaje receptivo/ expresivo (pruebas de vocabulario, de lenguaje receptivo y de tareas cognitivas), no teniendo en consideración otros aspectos lingüísticos como el fonológico, sintáctico, morfológico y pragmático, que son primordiales para la estructuración del habla y del lenguaje y de cuyo análisis obtendremos la información necesaria para intervenir en un área determinada. De las 74, la prueba más utilizada fue el PPVT-III Peabody en un $12 \%$ de estudios. Como contrapunto, Borg, Edquist, Reinholdson, Risberg y McAllister (2007), establecen una estructura teórica de la que parten para realizar la selección, basada en los tres componentes de los que depende la elaboración del lenguaje: el aferente, el central y el eferente. Consideran que la señal aferente es auditiva o visual y somatosensorial y la eferente consiste en el lenguaje oral o signado. Y así por ejemplo usan la "discriminación de fonemas" para valorar el sistema aferente, el PPVT-III Peabody (percepción de palabras) sería aferente y central, y la "producción de palabras" sería central y eferente.

Tal y como queda reflejado en los resultados de este trabajo, consideramos que en general, las pruebas seleccionadas son adecuadas para aplicar a los participantes con discapacidad auditiva de nuestra investigación, pero a través del análisis que obtengamos tras su administración, podremos definir su validez como instrumentos de evaluación de los diferentes componentes del lenguaje en la población de estudio. No obstante, resultaría muy beneficioso encontrar además una prueba que pudiera informar directamente de la capacidad cognitiva (de la memoria de trabajo y de la velocidad del procesamiento); porque la influencia de la hipoacusia y su posterior habilitación sobre el desarrollo del lenguaje dependen de una capacitación global, que cuando está por debajo de un determinado nivel de funcionamiento acarrea otros problemas lingüísticos añadidos (Borg et al., 2002; Borg et al., 2007). Hay un porcentaje de niños con hipoacusia, en los que, a pesar de aplicar la tecnología más avanzada, siguen manifestando retrasos significativos del lenguaje. En ocasiones se plantean dudas respecto a si la pérdida auditiva ha sido la causa de este retraso o si una perturbación concomitante es el factor fundamental. La afectación biológica de las funciones aferentes podría conducir a una degeneración trans-neuronal (Webster y Webster, 1977) que influyera en la maduración y desarrollo de las capacidades 
superiores, pero también sabemos que las alteraciones del procesamiento auditivo central son más frecuentes en caso de hipoacusia (Hansson, Forsberg, Löfqvist, Mäki-Torkko y Sahlén, 2004), por lo que necesitaríamos disponer de algún instrumento que nos permitiera detectar estas complejas diferencias.

\section{Conclusión:}

No existiendo consenso, consideramos que en el niño con hipoacusia es fundamental analizar cada uno de los aspectos del lenguaje, para tener un conocimiento más completo de su desarrollo lingüístico, que no es homogéneo en todos los ámbitos y se ve influenciado por otros factores, a veces muy difíciles de determinar. Las pruebas que hemos escogido permiten su aplicación clínica, aunque requerirían en algún caso, teniendo en cuenta las opiniones versadas por quienes las han aplicado así como por los profesionales logopedas consultados, adaptaciones específicas para esta población. En este trabajo, no obstante, y dado que nuestro propósito ha sido obtener valores psicométricos que nos permitan comparar los resultados de la población estudiada con la norma estadística, no se realizó ningún tipo de adaptación, siendo en todo momento rigurosos con las condiciones de aplicación que requiere la objetividad de este tipo de pruebas, a fin de no alterar la fiabilidad de las mismas.

\section{Agradecimientos:}

A todos los que participaron en las entrevistas realizadas. A los compañeros de logopedia (Grupo Serendipia de Lenguaje y Audición) por sus opiniones sobre la aplicación de las pruebas de lenguaje y a las logopedas profesionales por ofrecernos su tiempo y experiencia.

Este trabajo recibe la "Ayuda para el proyecto de investigación en Biomedicina seleccionado para desarrollar en los centros de la Gerencia Regional de Salud de Castilla y León en 2018. GRS 1497/A/17’.

\section{Bibliografía:}

Aguado-Alonso. G. (1989). El desarrollo de la morfosintaxis en el niño (TSA). $3^{\text {a }}$ Ed. Madrid, España: CEPE. Ciencias de la Educación Preescolar y Especial.

Aguinaga, G., Armentia, M.L., Fraile, A., Olangua, P., y Uriz, N. (2004). P.L.O.N.-R. Prueba de Lenguaje Oral de Navarra, Revisada. Madrid, España: TEA Ediciones.

American Academy of Pediatrics. Task Force on Newborn an Infant Hearing. (1999). Newborn and infant hearing loss: Detection and intervention. Pediatrics, 103, 527-30.

Benito-Orejas, J.I., Poncela-Blanco, M., García-Vicario, F., Benito-González, F., Martín-Sigüenza, G., y San Román-Carbajo, J. (2016). ¿Es fácil encargarse de coordinar un «programa de hipoacusia infantil»? Revista ORL, 7(2), 77-90.

Benito-Orejas, J.I., Ramírez, B., Morais, D., Fernández-Calvo, J.L., y Almaraz, A. (2008). Resultados de aplicar durante 42 meses un protocolo universal de detección e intervención precoz de la hipoacusia en neonatos. Acta Otorrinolaringológica Española, 59(3), 96-101. 
Benito-Orejas, J.I., Santiago-Pardo, R.B., Romero-Ureña, C., Sánchez-Rosso, A.L., Muñoz-Moreno, M.F., Escarda-Bolaños, M., et al. (2017). Metodología aplicada en el estudio del desarrollo del lenguaje en niños con detección temprana de la hipoacusia neonatal. Revista ORL, 8(2), 85-103.

Benito-Orejas, J.I. y Silva-Rico, J.C. (2013). Hipoacusia: Identificación e intervención precoces. Pediatría Integral, XVII(5), 330-42.

Borg, E., Edquist, G., Reinholdson, A.C., Risberg, A., y McAllister, B. (2007). Speech and language development in a population of Swedish hearing-impaired pre-school children, a cross-sectional study. International Journal of Pediatric Otorhinolaryngology, 71(7), 1061-77.

Borg, E., Risberg, A., McAllister, B., Undemar, B.M., Edquist, G., Reinholdson, A.C., et al. (2002). Language development in hearing-impaired children. Establishment of a reference material for a 'Language test for hearing-impaired children', LATHIC. International Journal of Pediatric Otorhinolaryngology, 65(1), 15-26.

Bosch-Galcerán, L. (2004). Evaluación fonológica del habla infantil. Barcelona, España: Masson.

Brancal, M.F., Alcantud, F., Ferrer, A.M., y Quiroga, M.E. (2009). EDAF: Evaluación de la discriminación auditiva y fonológica. $2^{\mathrm{a}}$ Ed. Barcelona, España: Ediciones Lebón S.L.

Calet-Ruiz, N., Mendoza-Lara, E., Carballo-García, G., Fresneda-López, M.D., y Muñoz-López, J. (2010). CEG 2-4 (test de comprensión de estructuras gramaticales de 2 a 4 años): estudio piloto. Revista de Logopedia, Foniatría y Audiología, 30(2), 62-72.

CODEPEH (Comisión para la detección precoz de la hipoacusia infantil). Trinidad-Ramos, G., Alzina, V., Jáudenes-Casaubón, C., Núñez Batalla, F., y Sequí-Canet, J.M. (2010). Recomendaciones de la CODEPEH para 2010. Acta Otorrinolaringológica Española, 61(1), 69-77.

Dunn, Ll.M., Dunn, L.M., y Arribas, D. (2010). PPVT-III PEABODY. Test de vocabulario en imágenes. Madrid, España: TEA Ediciones.

Equipo de Audición y Lenguaje del CREENA. (2006). Alumnado con grave discapacidad auditiva en Educación Infantil y Primaria. Orientaciones para la respuesta educativa. Fondo de Publicaciones del Gobierno de Navarra, $1^{a}$ Ed, pp 477.

Fitzpatrick, E., Angus, D., Durieux-Smith, A., Graham, I.D. y Coyle, D. (2008). Parent's needs following identification of childhood hearing loss. American Journal of Audiology, 17(1), 38-49.

García, J., Sánchez, M.L., y Delviso, S. (1986). Traducción al español del Test de Boston para el Diagnóstico de la Afasia. 2 ${ }^{\mathrm{a}}$ Ed. Madrid, España: Panamericana.

Gómez, P., Valero, I., Buades, R., y Pérez, A. (2005). THM. Test de Habilidades Metalingüísticas. Madrid, España: EOS (Instituto de Orientación Psicológica).

Goodglass, H., y Kaplan, E. (1986). La evaluación de la afasia y trastornos relacionados. 2a Ed. Madrid, España: Panamericana.

Gotzens, A., y Marro, S. (2001). Pruebas de valoración de la percepción auditiva: explorando los sonidos y el lenguaje. Barcelona, España: Masson.

Hansson, K., Forsberg, J., Löfqvist, A., Mäki-Torkko, E., y Sahlén, B. (2004). Working memory and novel word learning in children with hearing impairment and children with specific language impairment. International Journal of Language \& Communication Disorders, 39(3), 401-22.

Johnson, W., Darley, F.L., y Spriestersbach, D.C. (1963). Diagnostic Methods in Speech Pathology. New York: Harper \& Row.

Joint Committee on Infant Hearing. (2007). Year 2007 position statement: principles and 
guidelines for early hearing detection and intervention programs. American Academy of Pediatrics. Pediatrics, 120(4), 898-921.

Joint Committee on Infant Hearing. Position Statement. (1982). ASHA, 24, 1017-18.

Junta de Castilla y León. Consejería de Sanidad. (2004). Programa de detección precoz y atención integral de la hipoacusia infantil. Junta de Castilla y León. Consejería de Sanidad.

Kirk, S.A., McCarthy, J.J., y Kirk, W.D. (2004). Test de Illinois de Aptitudes psicolingüisticas (ITPA). Madrid, España: TEA Ediciones.

Kline, M., y Sapp, G.L. (1989). Carolina Picture Vocabulary Test: validation with hearing-impaired students. Percept Mot Skills, 69(1), 64-6.

Kral, A., y O'Donoghue, G. (2010). Profound deafness in chidhood. The New England Journal of Medicine, 363, 1438-50.

Lederberg, A.R., Schick, B., y Spencer, P.E. (2012). Language and literacy development of deaf and hard-of hearing children: Successes and challenges. Developmental Psichology, 30, 1-16.

López-Ginés, M.J., Redón-Díaz, A., Zurita-Salellas, M.D., García-Martínez, I., Santamaría-Marí, M., e Iniesta-Martiarena, J. (1997). ELCE (Evaluación del Lenguaje Comprensivo y Expresivo). Revista de Logopedia, Foniatría y Audiología, 17(4), 251-58.

López-Ginés, M.J., Redón-Díaz, A., Zurita-Salellas, M.D., García-Martínez, I., Santamaría-Marí, M., e Iniesta-Martiarena, J. (2007). Exploración del lenguaje comprensivo y expresivo. $4^{\mathrm{a}}$ Ed. ELCE. Madrid, España: CEPE. Ciencias de la Educación Preescolar y Especial.

Manrique, M., Cervera-Paz, F.J., Huarte, A., Martínez, I., Gómez, A., y Vázquez de la Iglesia, F. (2004). Audición y lenguaje en niños menores de 2 años tratados con implante coclear. Anales del Sistema Sanitario de Navarra, 27(3), 305-17.

Manrique-Rodríguez, M., y Huarte-Irujo, A. (2013). Organización de un programa de implantes cocleares. Acta Otorrinolaringológica Española, 64(1), 55-67.

Martínez, R., Benito, J.I., Condado, Ma .A., Morais, D., y Fernández-Calvo, J.L. (2003). Resultados de la aplicación del protocolo de detección precoz de la hipoacusia en neonatos de alto riesgo. Anales Otorrinolaringológicos Ibero-Americanos, XXX(3), 277-87.

Mayne, A.M., Yoshinaga-Itano, C., Sedey, A.L., y Carey, A. (2000). Expressive vocabulary development of infants and toddlers who are deaf or hard of hearing. The Volta Review, 100, 1-28.

Mendoza, E., Carballo, G., Muñoz, J., y Fresneda, M.D. (2005). CEG. Test de Comprensión de Estructuras Gramaticales. Madrid, España: TEA Ediciones.

Mohr, P.E., Feldman, J.J., Dunbar, J.L., McConkey-Robbins, A., Niparko, J.K., Rittenhouse, R.K., et al. (2000). The societal costs of severe to profound hearing loss in the United States. International Journal of Technology Assessment in Health Care, 16(4), 1120-35.

Molina-Hurtado, M., y Huarte-Irujo, A. (2003). Exploración del lenguaje. En: M. Manrique-Rodríguez, y A. Huarte-Irujo (Ed.), Implantes cocleares (pp. 145-148). Barcelona, España: Masson.

Monfort, M., y Juárez, A. (1989). Registro Fonológico Inducido. Madrid, España: CEPE. Ciencias de la Educación Preescolar y Especial.

Moog, J., Kozak, V.J., y Geers, A.E. (1987). Grammatical analysis of elicited language $(G A E L-P)$. Central Institute for the Deaf. Madrid, España: ECODA.

Northern, J.L., y Downs, M.P. (1981) La audición en los niños. Barcelona, España: Versión española de $2^{\mathrm{a}}$ ed. Salvat Editores, S.A.

Pérez, E., y Serra, M. (1998). A-REL. Análisis del Retraso del Lenguaje. Barcelona, España: Ariel. 
Puyuelo-Sanclemente, M., Renom-Pinsach, J., Solanas-Pérez, A., y Wiig, E.H. (2007). B.L.O.C,-S.R. Batería del Lenguaje Objetivo y Criterial -Screening Revisado. Barcelona, España: Masson.

Rico-Paino, M.I. (2016). Estudio analítico y observacional de las pruebas del lenguaje utilizadas en sujetos con discapacidad auditiva. Trabajo de Fin de Grado de Logopedia. Facultad de Medicina. Universidad de Valladolid.

Ruiz-Paredes, C. (2011). Atención temprana. Intervención logopédica. En: G. Trinidad y C. Jáudenes (Ed.), Sordera infantil. Del diagnóstico precoz a la inclusión educativa. Guía práctica para el abordaje interdisciplinar (pp. 163-176). Madrid, España: Confederación Española de Familias de Personas Sordas (FIAPAS).

Schroeder. L., Petrou, S., Kennedy, C., McCann, D., Law, C., Watkin, P.M., et al. (2006). The economic costs of congenital bilateral permanent childhood hearing impairment. Pediatrics, 117(4), 1101-12.

Silva, M.P., Comerlatto Junior, A.A., Bevilacqua, M.C., y Lopes-Herrera, S.A. (2011). Instruments to assess the oral language of children fitted with a cochlear implant: a systematic review. Journal of Applied Oral Science, 219(6), 549-53.

Svirsky, M.A. (2002). Efecto del implante coclear en el desarrollo lingüístico de niños con hipoacusia profunda prelocutiva. En: M. Manrique-Rodríguez y A. Huarte-Irujo (Ed.), Implantes cocleares (pp. 325-36). Barcelona, España: Masson.

Toro-Trallero, J., Cervera-Laviña, M., y Urío-Ruiz, C. (2002). E.M.L.E. Escala Magallanes de Lectura y Escritura. TALE 2000. Bilbao, España: Grupo ALBOR-COHS, Consultores de Ciencias Humanas, S.L.

Vohr, B., Jodoin-Krauzyk, J., Tucker, R., Johnson, M.J., Topol, D., y Ahlgren, M. (2008). Early language outcomes of early-identified infants with permanent hearing loss at 12 to 16 months of age. Pediatrics, 122(3), 535-44.

Webster, B.D., y Webster, M. (1977). Neonatal sound deprivation affects brainstem auditory nuclei. Archives of Otolaryngology-Head \& Neck Surgery, 103, 392-6.

Wechsler, D. (2005). WISC-IV. Escala de Inteligencia de Wechsler para niños-IV. Adaptación española. Madrid, España: TEA Ediciones.

Yoshinaga-Itano, C., Coulter, D.K., y Thomson, V. (2000). The Colorado Newborn Hearing Screening Project: effects on speech and language development for children with hearing loss. Journal of Perinatology, 20(suppl 8), S132-S137. 